\title{
Avaliação comportamental de ratos treinados em natação forçada
}

\author{
Behavioral assessment of rats through the forced-swimming test
}

\author{
Ana Luiza Peretti ${ }^{1}$, Camila Thieime Rosa ${ }^{2}$, Tatiane Kamada Errero', Vinícius Baretta1 \\ Sara Cristina Sagae Schneider ${ }^{3}$, Gladson Ricardo Flor Bertolini ${ }^{4} \bowtie$ \\ ${ }^{1}$ Estudante de graduação em Fisioterapia pela Universidade Estadual do Oeste do Paraná (Unioeste), Cascavel, PR. \\ 2 Fisioterapeuta formada pela Unioeste, Cascavel, PR. \\ 3 Doutora em Ciências Biológicas pela Universidade Federal do Rio Grande do Sul (UFRCS). Professora da Unioeste, Cascavel, PR. \\ ${ }^{4}$ Doutor em Ciências da Saúde Aplicadas ao Aparelho Locomotor pela Faculdade de Medicina de Ribeirão Preto da Universidade de São Paulo (FMRP/USP). \\ Professor da Graduação em Fisioterapia e do Mestrado em Biociências e Saúde da Unioeste, Cascavel, PR.
}

\section{RESUMO}

Objetivos: O exercício físico regular tem diversos efeitos benéficos, contudo, há relatos de aumento na concentração de corticosteroides endógenos, os quais podem inibir a neurogênese e piorar as funções cognitivas, sendo este um aspecto dúbio do exercício físico. $\mathrm{O}$ objetivo deste estudo foi avaliar, por meio de testes comportamentais, o efeito do exercício crônico por natação forçada com e sem sobrecarga, sobre parâmetros de ansiedade em ratos Wistar.

Métodos: Foram utilizados 24 ratos Wistar, separados em três grupos: Grupo Controle - submetido apenas a avaliações e contato com a água; Grupo Natação - composto por animais submetidos à natação com tempo progressivo; Grupo Natação com Sobrecarga - composto por animais submetidos à natação com sobrecarga de $10 \%$ do peso corporal. O exercício ocorreu três vezes por semana, no período vespertino, com aumento no tempo a cada duas semanas (20,30 e 40 minutos), totalizando seis semanas. Foi realizado em um tanque de água circular de 200 litros, com profundidade de $50 \mathrm{~cm}$ e temperatura da água mantida entre 30 e $32^{\circ} \mathrm{C}$. A ansiedade dos animais foi avaliada pelos testes Labirinto em Cruz Elevado e Campo Aberto.

Resultados: Não houve diferenças significativas em qualquer dos testes comportamentais, comparando os três grupos.

Conclusões: Não houve alteração no padrão comportamental de ratos Wistar submetidos a diferentes protocolos de exercício (natação forçada com e sem sobrecarga) quando comparados ao grupo controle não submetido a exercícios.

DESCRITORES: comportamento animal; exercício; estresse psicológico.

\section{ABSTRACT}

Aims: Regular exercise has several beneficial effects. However, reports show increasing concentrations of endogenous corticosteroids, which may inhibit neurogenesis and deteriorate cognitive functions, thus raising doubts as to the benefits of physical exercise. The objective of this study was to evaluate, through behavioral tests, the effect of chronic forced swimming with and without overload on anxiety parameters in Wistar rats.

Methods: Twenty-four Wistar rats, divided into three groups, were assessed: Control Group - subjected only to evaluations and contact with water; Swimming Group - consisting of animals submitted to progressive swimming time; Weight-loaded Swimming Group - consisting of animals submitted to swimming with loads in excess of $10 \%$ of their body weight. The exercise was performed three times a week in the afternoon, with swimming time increases every two weeks $(20,30$, and 40 minutes), during six weeks, A 200-liter circular water tank, filled to a depth of $50 \mathrm{~cm}$, was used, and the water temperature was kept between $30^{\circ}$ and $32^{\circ} \mathrm{C}$. The animals' anxiety was assessed by the Elevated Plus Maze and Open Field tests.

Results: No significant differences between the three groups were detected by any of the behavioral tests.

Conclusions: No changes were observed in the behavioral pattern of Wistar rats submitted to different exercise protocols (forced swimming with and without overload) compared to the control group.

KEY WORDS: behavior, animal; exercise; stress, psychological. 


\section{INTRODUÇÃO}

O exercício físico regular apresenta benefícios tanto para a prevenção quanto para o tratamento de doenças, como hipertensão arterial, resistência à insulina, diabetes, dislipidemia e obesidade, além de melhora cognitiva. Observou-se, também, que em indivíduos acima de 60 anos a prática de exercício regular reduz a depressão e a ansiedade [1-4].

Em estudo com ratos, o exercício físico de baixa intensidade mostrou-se como um atenuador de alterações tanto de insulina e metabólitos da corticosterona, quanto do comportamento alimentar promovido pelo estresse social induzido pelo isolamento, com provável ação sobre o eixo hipotalâmico-pituitárioadrenal [5]. Observou-se que o exercício retarda o desenvolvimento de neurite autoimune em ratos [6]. Contudo, de forma contraditória, há relatos sobre elevação nas concentrações de corticosterona e cortisol [7-9].

O exercício pode ser utilizado como agente para produzir a redução de quadros álgicos, sendo que sua provável ação é devida à liberação de opioides endógenos, tanto central quanto perifericamente [10-13]. Ainda, possui ações anti-inflamatórias por ativação imune, que culminam em eventos antidepressivos [14,15]. Contudo, em animais submetidos a modelo experimental agudo de dor articular em joelhos, a natação por 10 minutos não produziu diminuição do quadro álgico [16], provavelmente por causa da pequena duração e intensidade do exercício, visto que, segundo Koltyn e Umeda [17], a hipoalgesia parece ocorrer quando o exercício é realizado em altas intensidades. Ainda, observaram, em camundongos, que a corrida voluntária em esteira gerou aumento na ansiedade, avaliado pelo teste de campo aberto, labirinto elevado e caixa claro/escuro, além de altos níveis de corticosterona nas fezes dos animais [18].

Existem evidências de que o exercício pode aumentar a neurogênese, melhorar a função cognitiva e proteger o cérebro contra distúrbios neurodegenerativos. Contudo, em animais que realizam caminhada em esteira do tipo forçado, os níveis de corticosterona podem aumentar, o que subsequentemente regularia para baixo a neurogênese e pioraria a função cognitiva [19]. Desta forma, o exercício físico é utilizado tanto visando redução de quadros de dor [17] quanto de ansiedade e depressão [4]. Assim sendo, considerando as controvérsias, o objetivo deste estudo foi avaliar, por meio de testes comportamentais, o efeito da natação forçada crônica, sem e com sobrecarga, sobre parâmetros de ansiedade em ratos.

\section{MÉTODOS}

Foram utilizados 24 ratos Wistar, machos, com $10 \pm 2$ semanas de idade, obtidos no Biotério Central da Unioeste, e mantidos, no Biotério Setorial do Laboratório de Estudo das Lesões e Recursos Fisioterapêuticos, em caixas de polipropileno, com livre acesso a água e ração ad libitum, ciclo claro/ escuro controlado de 12 horas e temperatura ambiente controlada $\left(24 \pm 1^{\circ} \mathrm{C}\right)$. O estudo foi conduzido segundo as normas internacionais de ética em experimentação animal, sendo aprovado pelo Comitê de Ética em Uso Animal da Unioeste, certificado sob o número 15/2014-CE.

Os animais foram separados aleatoriamente em três grupos: Grupo Controle ( $\mathrm{GC}, \mathrm{n}=8)$ - submetido apenas às avaliações e contato com água; Grupo Natação $(\mathrm{GN}, \mathrm{n}=8)$ - composto por animais submetidos à natação com tempos progressivos; e Grupo Natação com Sobrecarga (GNS, $\mathrm{n}=8$ ) - composto por animais submetidos à natação com sobrecarga de $10 \%$ do peso corporal.

O GN foi submetido a protocolo de exercícios, três vezes por semana, com tempos crescentes a cada duas semanas $(20,30$ e 40 minutos $)$, totalizando seis semanas de natação. Foi utilizado um tanque de água de 200 litros, circular, com profundidade de 50 $\mathrm{cm}$, sendo a temperatura da água mantida entre $30^{\circ}$ e $32^{\circ} \mathrm{C}$. O exercício ocorreu no período vespertino. O GNS recebeu sobrecarga corporal de $10 \%$, com pesos de chumbo unidos a uma tira de velcro na região abdominal dos animais, o que não interferiu na natação, sendo que os animais eram pesados sempre previamente ao exercício. Os animais do GC eram colocados no ambiente aquático mas permaneciam por somente 10 segundos, sendo retirados ao final desse tempo, visando a que experimentassem apenas o estresse da água, porém sem o do exercício.

Os testes comportamentais foram realizados, no período vespertino, aproximadamente 24 horas após a última sessão de exercícios. A ansiedade dos animais foi avaliada pelos testes Campo Aberto [20] e Labirinto em Cruz Elevado [21], nesta sequencia. No teste do Campo Aberto (Open Field) foi utilizada uma arena dividida em 12 quadrantes. $\mathrm{O}$ animal era colocado na porção central e as seguintes variáveis comportamentais eram registradas durante 5 minutos: período de imobilidade; número de ambulações na periferia (áreas com paredes laterais); número de ambulações no centro (áreas sem contato com paredes laterais); número de elevações das patas traseiras (rearings) e tempo de autolimpeza (grooming) [22,23]. 
Os dados foram analisados quanto à sua normalidade por meio do teste de Kolmogorov-Smirnov e, posteriormente, foi utilizado o teste de ANOVA unidirecional para comparação dos grupos. O nível de significância aceito foi de 5\%.

\section{RESULTADOS}

A variação entre as análises feitas pelos dois investigadores foi inferior a 5\%.

Os resultados não mostraram diferenças significativas em nenhum dos testes realizados, ao comparar os três grupos de ratos (Tabela 1).

\section{DISCUSSÃO}

O presente estudo buscou avaliar o comportamento de ratos Wistar, submetidos à natação forçada, em variáveis de comportamentos de ansiedade. Verificou-se que, independente do tipo e intensidade do exercício, não houve alteração no padrão comportamental dos mesmos em ambos os testes utilizados. Considerando que um aumento na ambulação central (número de cruzamentos) indica redução da ansiedade [24], enquanto que maior exploração de braços fechados em comparação aos abertos é interpretada como maior ansiedade, os resultados mostram que o exercício físico não foi capaz de induzir uma alteração consistente na ansiedade dos animais avaliados.

Tal fato difere de um estudo realizado em humanos, no qual foi possível observar que tanto o exercício de resistência quanto o resistido podem produzir aumento nos níveis de cortisol, o que provavelmente favorece processos anabólicos dentro do músculo, regulando o metabolismo da glicose e do glicogênio, sendo que o estresse ao exercício pode ser a razão deste tipo de resposta [25]. A esse estresse também pode ser creditado o efeito analgésico do exercício [16]. Apesar de haver grande semelhança fisiológica, celular e molecular entre os modelos animais e humanos, existem limitações no uso de animais, e a extrapolação de resultados de modelos para humanos deve ser realizada com cautela.

Não é possível deixar de considerar, ainda, que os testes de Campo Aberto e Labirinto em Cruz Elevado, apesar de serem amplamente utilizados em estudos de comportamento dos animais, apresentam limitações e variações, o que poderia talvez explicar parcialmente a ausência de efeito do exercício físico sobre os parâmetros comportamentais avaliados no presente estudo. Exemplos incluem o tempo e as condições de iluminação no teste de Campo Aberto, e a reduzida sensibilidade do Labirinto em Cruz Elevado ao efeito ansiolítico de alguns antidepressivos. A grande maioria dos modelos animais de ansiedade produziram perfis inconsistentes e muitas vezes contraditórios para agonistas serotoninérgicos [26,27]. Estudos mostram resultados também contraditórios na análise dos efeitos de ansiolíticos, como benzodiazepínicos, injetados em ratos, podendo aumentar o número de entradas e o tempo gasto nos braços abertos do labirinto [28], ou podendo reduzi-lo [27]. Adicionalmente, apesar de

Tabela 1. Apresentação dos comportamentos evocados por 24 ratos Wistar, machos, com $10 \pm 2$ semanas de idade, nos testes Labirinto em Cruz Elevado e Campo Aberto, de acordo com os diferentes grupos avaliados. Os dados são expressos como média \pm desvio-padrão da média do tempo em minutos em que o comportamento foi observado.

\begin{tabular}{|c|c|c|c|c|}
\hline Comportamento & GC & GN & GNS & $\mathbf{p}^{*}$ \\
\hline \multicolumn{5}{|l|}{ Teste do Labirinto em Cruz Elevado } \\
\hline Tempo no braço fechado & $3,35 \pm 4,52$ & $3,68 \pm 6,47$ & $1,48 \pm 3,16$ & 0,7052 \\
\hline Tempo no braço aberto & $51,76 \pm 18,42$ & $65,16 \pm 27,15$ & $60,91 \pm 28,22$ & 0,5958 \\
\hline Tempo no braço fechado olhando para fora & $12,06 \pm 10,91$ & $12,15 \pm 18,95$ & $10,49 \pm 9,35$ & 0,9724 \\
\hline Tempo no braço aberto olhando para fora & $11,52 \pm 5,98$ & $11,53 \pm 7,50$ & $11,42 \pm 12,84$ & 0,9997 \\
\hline \multicolumn{5}{|l|}{ Teste do Campo Aberto } \\
\hline Tempo andando na lateral & $40,70 \pm 21,77$ & $31,07 \pm 13,34$ & $28,29 \pm 18,81$ & 0,4457 \\
\hline Tempo parado & $44,86 \pm 24,68$ & $54,18 \pm 26,78$ & $53,32 \pm 29,22$ & 0,7788 \\
\hline Tempo andando no centro & $1,19 \pm 1,57$ & $1,12 \pm 1,01$ & $0,83 \pm 1,44$ & 0,8856 \\
\hline Número de elevações das patas traseiras (rearing) & $10,42 \pm 5,55$ & $5,20 \pm 2,16$ & $5,38 \pm 8,14$ & 0,1828 \\
\hline Tempo de autolimpeza (grooming) & $6,24 \pm 3,01$ & $7,69 \pm 4,99$ & $12,08 \pm 12,98$ & 0,4053 \\
\hline
\end{tabular}

GC (Grupo Controle): submetido apenas a contato com a água; GN (Grupo Natação): submetido à natação com tempo progressivo; GNS (Grupo Natação com Sobrecarga): submetido à natação com sobrecarga de $10 \%$ do peso corporal.

* ANOVA unidirecional. 
não haver dúvida de que o Labirinto em Cruz Elevado é altamente sensível à influência de manipulações relacionadas com o receptor benzodiazepínico $\mathrm{GABA}_{\mathrm{A}}$, os efeitos obtidos com outros agentes moduladores de ansiedade têm sido muito mais variáveis [26].

Andersen et al. [29] observaram, em ratos, que a natação forçada, duas vezes por dia, uma hora por sessão, por quatro dias, não foi capaz de produzir estresse suficiente para gerar aumento nos níveis de corticosterona. Apesar de no presente estudo não ter sido realizada a dosagem hormonal, tal resultado é compatível com o que foi observado nos testes comportamentais, indicando que mesmo a natação forçada com sobrecarga foi insuficiente para produzir alterações na ansiedade dos animais. Já Gavrilovic et al. [30] observaram que ratos estressados por isolamento social e que realizaram exercício em esteira por 12 semanas (iniciando com 10 minutos na velocidade de 10 metros por minuto e aumentando até 20 minutos na velocidade de 20 metros por minuto), não apresentaram redução nas concentrações de catecolaminas e corticosterona plasmáticas, nem hipertrofia adrenal. Ainda, Fuss et al. [18] relatam que em camundongos exercitados voluntariamente, por corrida em esteira, apesar de maiores concentrações de Fator Neurotrófico Derivado do Cérebro (BDNF, Brain-Derived Neurotrophic Factor) e neurogênese no hipocampo, apresentaram aumento no comportamento de ansiedade e maiores níveis de corticosterona (indicativos de estresse) em suas fezes.

De forma contraditória, Calik et al. [6] relatam que em ratos adultos submetidos a modelo experimental de síndrome de Guillain-Barré, o exercício forçado (corrida em esteira 1,2 km por dia, durante três semanas) produziu efeito protetor, com relação aos déficits nervosos periféricos, tendo assim efeitos antiinflamatórios, com redução nos níveis plasmáticos de corticosterona total. Assim também, Filipovic et al. [31] observaram que ratos que realizavam natação diária, por 15 minutos, apresentaram diminuição dos efeitos do isolamento social crônico sobre o eixo hipotalâmico-hipofisário-adrenal. Patki et al. [32] observaram, também em ratos submetidos a modelo de estresse pós-traumático, que um protocolo diário de 30 minutos de exercício em esteira ( $1^{\text {a }}$ semana 10 metros por minuto; $2^{\mathrm{a}}$ semana -15 metros por minuto) produziu alívio tanto nos sintomas de ansiedade quanto nos comportamentos depressivos, assim como estresse e déficits cognitivos, com diminuição dos níveis de corticosterona plasmáticos.

Waters et al. [33] dividiram dois grupos de ratos de acordo com suas características familiares, com objetivo de avaliar a se as características intrínsecas de aptidão para o exercício teriam influência sobre o estresse. Observaram que os animais com alta capacidade ao exercício de resistência exibiram maior comportamento de ansiedade do que os animais com baixa capacidade para o exercício, e ainda exibiram maiores níveis de corticosterona [33]. Contudo, Martins e Lopes [34], avaliando militares brasileiros, observaram associação do estresse devido ao trabalho e do sofrimento psicológico (identificado por sintomas de depressão e nervosismo) com baixos níveis de atividade física na prática de esportes/exercícios.

O exercício físico tem sido testado para aliviar diversos tipos de estresse experimentalmente induzidos, como isolamento social [5,30], ruídos [35], imobilização, frio [31], privação de alimentos e água [14], dentre outros. Há indícios de que o tipo e a intensidade do exercício, bem como o modelo animal utilizado, podem influenciar nas respostas hormonais e comportamentais [36].

Como limitação do presente estudo pode-se considerar o desconhecimento dos níveis de corticosterona no plasma ou nas fezes, que poderiam contribuir para uma análise mais profunda dos efeitos do exercício sobre o estado de ansiedade/depressão. Contudo, ressalta-se que os testes comportamentais escolhidos são bastante utilizados em pesquisa e foram aplicados com rigor científico, o que permite concluir que os protocolos de exercício usados (natação forçada com e sem sobrecarga) não produziram alterações comportamentais nos animais sob as condições em que este experimento foi realizado.

\section{REFERÊNCIAS}

1. Ciolac EG, Guimarães GV. Exercício físico e síndrome metabólica. Rev Bras Med Esporte. 2004;10(4):319-24. http://dx.doi.org/10.1590/ S1517-86922004000400009

2. Liebetanz D, Gerber J, Schiffner C, Schütze S, Klinker F, Jarry H, et al. Pre-infection physical exercise decreases mortality and stimulates neurogenesis in bacterial meningitis. J Neuroinflammation. 2012;9:168. http://dx.doi.org/10.1186/1742-2094-9-168

3. Langlois F, Tuong T, Vu M, Chassé K, Dupuis G, Kergoat M, et al. Benefits of physical exercise training on cognition and quality of life in frail older adults. J Gerontol B Psychol Sci Soc Sci. 2012;68(3):400-4. http://dx.doi.org/10.1093/geronb/gbs069 
4. Cheik NC, Reis IT. Efeitos do exercício físico e da atividade física na depressão e ansiedade em indivíduos idosos. R Bras Ci e Mov. 2003;11(3):45-51.

5. Benite-Ribeiro SA, Matzenbacher J, Duarte JAR. Moderate physical exercise attenuates the alterations of feeding behaviour induced by social stress in female rats. Cell Biochem Funct. 2014;32(4):142-9. http://dx.doi.org/10.1002/cbf.2984

6. Calik MW, Shankarappa SA, Stubbs EB. Forced-exercise attenuates experimental autoimmune neuritis. Neurochem Int. 2012;61(2):141-5. http://dx.doi.org/10.1016/j.neuint.2012.04.023

7. Di Luigi L, Botrè F, Sabatini S, Sansone M, Mazzarino M, Guidetti L, Baldari C, Lenzi A, Caporossi D, Romanelli F, Sgrò P. Acute effects of physical exercise and phosphodiesterase's type 5 inhibition on serum 11b-hydroxysteroid dehydrogenases related glucocorticoids metabolites: a pilot study. Endocrine. 47(3):952-8. http://dx.doi.org/10.1007/s12020-014-0185-2

8. Navarro F, Bacurau AVN, Almeida SS, Barros CC, Moraes MR, Pesquero JL, Ribeiro SM, Araújo RC, Costa Rosa LF, Bacurau RF. Exercise prevents the effects of experimental arthritis on the metabolism and function of immune cells. Cell Biochem Funct. 2010;28(4):266-73 http://dx.doi.org/10.1002/cbf.1647

9. Cherem EHL, Santos LC dos, Azeredo FP de, Serra RA, Sá CCNF de. Alteração da testosterona, cortisol, força e massa magra após 20 semanas como resposta a três metodologias de treinamento de força. Rev Bras Fisiol Ex. 2014;13(4):188-96.

10. Kuphal KE, Fibuch EE, Taylor BK. Extended swimming exercise reduces inflammatory and peripheral neuropathic pain in rodents. J Pain. 2007;8(12):989-97. http://dx.doi.org/10.1016/j.jpain.2007.08.001

11. Bertolini GRF, Rosa CT, Silva LI, Meireles A, Rocha BP. Uso do exercício resistido antagonizado por naloxone como fator de analgesia em sinovite aguda de joelho de ratos Wistar. Rev Bras Med Esporte. 2012;18(2):126-9. http://dx.doi.org/10.1590/S1517-86922012000200013

12. Bement MKH, Sluka KA. Low-intensity exercise reverses chronic muscle pain in the rat in a naloxone-dependent manner. Arch Phys Med Rehabil. 2005;86(9):1736-40. http://dx.doi.org/10.1016/j.apmr.2005.03.029

13. Hua S, Cabot PJ. Mechanisms of peripheral immune-cell-mediated analgesia in inflammation: clinical and therapeutic implications. Trends Pharmacol Sci. 2010;31(9):427-33. http://dx.doi.org/10.1016/j.tips.2010.05.008

14. Liu W, Sheng H, Xu Y, Liu Y, Lu J, Ni X. Swimming exercise ameliorates depression-like behavior in chronically stressed rats: Relevant to proinflammatory cytokines and IDO activation. Behav Brain Res. 2013;242:110-6. http://dx.doi.org/10.1016/j.bbr.2012.12.041

15. Liu W, Xu Y, Lu J, Zhang Y, Sheng H, Ni X. Swimming exercise ameliorates depression-like behaviors induced by prenatal exposure to glucocorticoids in rats. Neuroscie Lett. 2012;524(2):119-23. http://dx.doi.org/10.1016/j.neulet.2012.07.011

16. Bertolini GRF, Matos CMP, Artifon EL, Ferrari D, Vituri RF. Avaliação funcional da nocicepção do joelho de ratos tratada com laser de baixa potência e natação. Rev Bras Med Esporte. 2011;17(1):45-8. http://dx.doi.org/10.1590/S1517-86922011000100009

17. Koltyn KF, Umeda M. Exercise, hypoalgesia and blood pressure. Sport Med. 2006;36(3):207-14. http://dx.doi.org/10.2165/00007256200636030-00003

18. Fuss J, Abdallah NMB, Vogt MA, Touma C, Pacifici PG, Palme R, Witzemann V, Hellweg R, Gass P. Voluntary exercise induces anxietylike behavior in adult C57BL/6J mice correlating with hippocampal neurogenesis. Hippocampus. 2010;20(3):364-76. http://dx.doi. org/10.1002/hipo.20634

19. Li H, Liang A, Guan F, Fan R, Chi L, Yang B. Regular treadmill running improves spatial learning and memory performance in young mice through increased hippocampal neurogenesis and decreased stress. Brain Res. 2013;1531:1-8. http://dx.doi.org/10.1016/j. brainres.2013.07.041

20. Walsh RN, Cummins RA. The open field test: a critical review. Psychol Bull. 1976;83(3):482-504. http://dx.doi.org/10.1037/00332909.83.3.482

21. Pellow S, Chopin P, File SE, Briley M. Validation of open:closed arm entries in an elevated plus-maze as a measure of anxiety in the rat. J Neurosci Methods. 1985;14(3):149-67. http://dx.doi.org/10.1016/0165-0270(85)90031-7

22. Pietá Dias C, Martins de Lima MN, Presti-Torres J, Dornelles A, Garcia VA, Siciliani Scalco F, Rewsaat Guimarães M, Constantino L, Budni P, Dal-Pizzol F, Schröder N. Memantine reduces oxidative damage and enhances long-term recognition memory in aged rats. Neuroscience. 2007;146(4):1719-25. http://dx.doi.org/10.1016/j.neuroscience.2007.03.018

23. De Lacerda GFM. Ansiedade em modelos animais: efeito de drogas nas dimensões extraídas da análise fatorial [dissertation]. [Curitiba]: Universidade Federal do Paraná; 2006. 62 p. Available from: http://dspace.c3sl.ufpr.br:8080/dspace/handle/1884/3780

24. Choleris E, Thomas AW, Kavaliers M, Prato FS. A detailed ethological analysis of the mouse open field test: effects of diazepam, chlordiazepoxide and an extremely low frequency pulsed magnetic field. Neurosci Biobehav Rev. 2001;25(3):235-60. http://dx.doi. org/10.1016/S0149-7634(01)00011-2

25. Smilios I, Pilianidis T, Karamouzis M, Tokmakidis SP. Hormonal responses after various resistance exercise protocols. Med Sci Sport Exerc. 2003;35(4):644-54. http://dx.doi.org/10.1249/01.MSS.0000058366.04460.5F

26. Handley SL, McBlane JW. 5HT drugs in animal models of anxiety. Psychopharmacology. 1993;112(1):13-20. http://dx.doi.org/10.1007/ BF02247358

27. Bertoglio LJ, Carobrez AP. Previous maze experience required to increase open arms avoidance in rats submitted to the elevated plus-maze model of anxiety. Behav Brain Res. 2000;108(2):197-203. http://dx.doi.org/10.1016/S0166-4328(99)00148-5

28. File SE, Zangrossi Jr H. "One-trial tolerance" to the anxiolytic actions of benzodiazepines in the elevated plus-maze, or the development of a phobic state? Psychopharmacology (Berl). 1993;110(1-2):240-4. http://dx.doi.org/10.1007/BF02246980

29. Andersen ML, Bignotto M, Machado RB, Tufik S. Different stress modalities result in distinct steroid hormone responses by male rats. Braz J Med Biol Res. 2004;37(6):791-7. http://dx.doi.org/10.1590/S0100-879X2004000600003 
30 Gavrilovic L, Stojiljkovic V, Kasapovic J, Popovic N, Pajovic SB, Dronjak S. Treadmill exercise does not change gene expression of adrenal catecholamine biosynthetic enzymes in chronically stressed rats. An Acad Bras Cienc. 2013;85(3):999-1012. http://dx.doi.org/10.1590/ S0001-37652013005000041

31. Filipovic D, Gavrilovic L, Dronjak S, Radojcic MB. The effect of repeated physical exercise on hippocampus and brain cortex in stressed rats. Ann N Y Acad Sci. 2007;1096:207-19. http://dx.doi.org/10.1196/annals.1397.087

32. Patki G, Li L, Allam F, Solanki N, Dao AT, Alkadhi K, et al. Moderate treadmill exercise rescues anxiety and depression-like behavior as well as memory impairment in a rat model of posttraumatic stress disorder. Physiol Behav. 2014;130:47-53. http://dx.doi.org/10.1016/j. physbeh.2014.03.016

33. Waters RP, Renner KJ, Summers CH, Watt MJ, Forster GL, Koch LG, et al. Selection for intrinsic endurance modifies endocrine stress responsiveness. Brain Res. 2010;1357:53-61. http://dx.doi.org/10.1016/j.brainres.2010.07.078

34. Martins LCX, Lopes CS. Rank, job stress, psychological distress and physical activity among military personnel. BMC Public Health. 2013;13:716. http://dx.doi.org/10.1186/14712458-13-716

35. Sasse SK, Greenwood BN, Masini C, Nyhuis TJ, Fleshner M, Day HEW, et al. Chronic voluntary wheel running facilitates corticosterone response habituation to repeated audiogenic stress exposure in male rats. Stress. 2008;11(6):425-37. http://dx.doi. org/10.1080/10253890801887453

36. Liu W, Zhou C. Corticosterone reduces brain mitochondrial function and expression of mitofusin, BDNF in depression-like rodents regardless of exercise preconditioning. Psychoneuroendocrinology. 2012;37(7):1057-70. http://dx.doi.org/10.1016/j.psyneuen.2011.12.003 